\title{
360-degree suture trabeculotomy ab interno to treat open-angle glaucoma: 2-year outcomes
}

This article was published in the following Dove Press journal: Clinical Ophthalmology

\section{Tomoki Sato' \\ Takahiro Kawaji' \\ Akira Hirata ${ }^{2}$ \\ Takanori Mizoguchi ${ }^{3}$}

'Sato Eye and Internal Medicine Clinic, Arao City, Kumamoto, Japan; ${ }^{2}$ Hayashi Eye Hospital, Hakata-ku, Fukuoka, Japan; ${ }^{3}$ Mizoguchi Eye Clinic, Sasebo, Nagasaki, Japan
Correspondence: Tomoki Sato Sato Eye and Internal Medicine Clinic, 4I60-270 Arao, Arao City, Kumamoto 864-004I, Japan

$\mathrm{Tel}+8 \mid 968655900$

Fax+8I 968655902

Email zxsato@gmail.com
Purpose: The purpose of this study was to evaluate the efficacy of 360-degree suture trabeculotomy (360S-LOT) ab interno for treating open-angle glaucoma (OAG). Risk factors of surgical failure were examined.

Patients and methods: 360S-LOT ab interno alone was performed for patients with uncontrolled OAG, and combined 360S-LOT ab interno/phacoemulsification was performed for patients with controlled OAG with a visually significant cataract between March 2014 and September 2015 at a single center. The patients were prospectively followed for 2 years. The main outcome measures included 2-year intraocular pressure (IOP), number of anti-glaucoma medications used, postoperative complications, and predictive factors of surgical failure. KaplanMeier analysis was performed, with surgical success (with or without medication use) defined as postoperative IOP $\leq 15 \mathrm{mmHg}$ and IOP reduction $\geq 20 \%$ (criterion A) or IOP $\leq 12 \mathrm{mmHg}$ and IOP reduction $\geq 30 \%$ (criterion $B$ ). Predictive factors were evaluated using Cox proportional hazard ratios.

Results: A total of 64 eyes of 64 patients were included, and 50 (78\%) eyes of 64 eyes underwent a phacoemulsification combination procedure. Surgery significantly reduced IOP from $18.4 \pm 2.9 \mathrm{mmHg}$ before surgery to $13.4 \pm 3.0 \mathrm{mmHg}$ after surgery $(P<0.001)$. Patients used an average of $1.8 \pm 1.5$ medications before surgery and $1.3 \pm 1.5$ medications after surgery $(P=0.101)$. No serious postoperative complications were observed. The probability of surgical success was $49.2 \%$ and $16.0 \%$ using criteria A and B, respectively. No risk factors of surgical failure were identified.

Conclusion: The 360S-LOT ab interno procedure is a favorable option for treating eyes with mild or moderate OAG.

Keywords: 360-degree suture trabeculotomy ab interno, open-angle glaucoma, minimally invasive glaucoma surgery, phacoemulsification

\section{Introduction}

Trabeculotomy lowers intraocular pressure (IOP) by reducing aqueous outflow resistance via 120 -degree incision that is made in the trabecular meshwork and Schlemm's canal inner layer (without bleb formation). ${ }^{1-3}$ Unfortunately, trabeculotomy alone does not generally allow IOP to be maintained at $<15 \mathrm{mmHg}$ after surgery, although IOP is further reduced when trabeculotomy is combined with cataract surgery. ${ }^{4-6}$

A modified 360-degree suture trabeculotomy (360S-LOT) ${ }^{7}$ involves making an incision along the entire circumference of the trabecular meshwork and Schlemm's canal inner layer using 5-0 nylon suture. This procedure has a possibility to lower IOP significantly more than standard trabeculotomy. ${ }^{7,8}$ Since the resistance of the Schlemm's canal inner layer disappears upon circumferential incision, the postoperative IOP should theoretically be close to the episcleral venous pressure, which was reported to 
be $\sim 12 \mathrm{mmHg}$ in both primary open-angle glaucoma (POAG) and normal tension glaucoma (NTG). ${ }^{9}$

We previously developed the 360S-LOT ab interno procedure that can preserves the conjunctiva and sclera, from a modified 360S-LOT ab externo technique, and analyzed short-term surgical safety and efficacy. ${ }^{10}$ Although the 360SLOT ab interno procedure was found to be both safe and effective, mid-term outcomes and risk factors of surgical failure have not yet been examined. The current study prospectively examines the 2-year efficacy of the 360S-LOT $\mathrm{ab}$ interno procedure and investigates the factors that may contribute to surgical failure in patients with open-angle glaucoma $(\mathrm{OAG})$.

\section{Patients and methods}

This prospective, nonrandomized, open-label, single-center, clinical trial was conducted at the Sato Eye and Internal Medicine Clinic (Arao City, Japan). All patients were enrolled between March 2014 and September 2015. The study protocol was reviewed and approved by the institutional review board and the ethics committee of Sato Eye and Internal Medicine Clinic. All study conduct adhered to the tenets of the Declaration of Helsinki, and written informed consent was obtained from all patients before any study examinations or procedures were performed.

\section{Study patients}

Patients were considered for enrollment in the 360S-LOT ab interno alone procedure if they had uncontrolled OAG with maximally tolerated anti-glaucoma medications. Furthermore, patients were considered for enrollment in the combined 360S-LOT ab interno/phacoemulsification with intraocular lens implant (PEA + IOL) procedure if they wished to undergo the combined procedure for a visually significant cataract that coexisted with controlled or uncontrolled glaucoma. This included patients with POAG, NTG, and exfoliation glaucoma (XFG). A cataract was considered to be visually significant if the patient complained of glare or halos and best-corrected visual acuity (BCVA) was 20/20 or worse. A POAG diagnosis was given if IOP was $>21 \mathrm{mmHg}$, the angle was open (Shaffer grade 3 or 4), optic neuropathy was present, and typical matching disk and field changes had occurred. An NTG diagnosis was given using the same criteria, but IOP was $\leq 21 \mathrm{mmHg}$. An XFG diagnosis was given if exfoliation material had adhered to the lens surface or the iris-pupil margin, the angle was wide open in the presence of dense pigmentation, Sampaolesi's line was present, and optic neuropathy with matching visual field loss had occurred. Patients were excluded from this study if they exhibited types of glaucoma other than POAG, NTG, or XFG or if they had a previous history of ocular trauma or glaucoma surgery. Patients were also excluded if they demonstrated the presence of other preexisting ocular diseases, a corneal abnormality that precluded reliable applanation tonometry, or the presence of a single functional eye.

\section{S-LOT ab interno procedure}

All procedures were performed by a single surgeon (T.S.). Only one eye of each patient was included in this study. In some cases, patients did require bilateral surgery, but only the first eye operated on was included in analyses. The 360S-LOT $\mathrm{ab}$ interno procedure has been previously described in full. ${ }^{10}$ Briefly, a 1.7-mm temporal cornea incision was created and Schlemm's canal was incised at $15^{\circ}$ on the nasal side using the Trabectome (NeoMedix Inc., Tustin, CA, USA). The rounded tip of a 5-0 nylon suture was then inserted into Schlemm's canal using 23-gage disposable forceps (Grieshaber Revolution ${ }^{\circledR}$ DSP $^{\circledR}$; Alcon Laboratories, Inc., Fort Worth, TX, USA). After passing the suture tip around the circumference of Schlemm's canal, the suture was pulled out through the same opening. The same forceps were then used to make a 360-degree incision in Schlemm's canal. The scleral wound integrity was assessed by closely monitoring for leakage. If necessary, standard phacoemulsification with PEA + IOL was performed following the 360S-LOT ab interno procedure through the same or a newly created upper corneal incision. Ocular integrity was again assessed via wound leakage examination.

\section{Study examinations}

All potential patients were screened for study eligibility using slit-lamp biomicroscopy, indirect ophthalmoscopy, manifest refraction, IOP measurement, corneal endothelial cell density (cells $/ \mathrm{mm}^{2}$ ), and BCVA assessment (conventional Landolt ring chart). IOP was measured using a Goldmann tonometer. Baseline IOP was determined by the mean of the three most recent measurements, acquired on separate days within 1 month of surgery. Corneal endothelial cell density was examined using a noncontact specular microscope (SP-3000P; Topcon, Tokyo, Japan). Manifest refraction was measured using an auto ref-keratometer (ARK-530A; Nidek, Tokyo, Japan); all decimal visual acuity values were converted to the logarithm of the minimum angle of resolution ( $\log$ MAR) for analyses.

All patients were examined on postoperative days 1, 2, and 3 . Thereafter, patients were examined every $1-2$ weeks until Month 1, monthly until Month 6, and every 2-3 months 
until Month 24. The IOP was measured at every study visit. Patients were administered anti-glaucoma medications following surgery if IOP was higher than the desired range $(<15 \mathrm{mmHg})$, as in the previous report. ${ }^{10}$

\section{Outcome measures}

Outcome measures were IOP at each study visit, the number of glaucoma medications used, cumulative surgical success rates (Kaplan-Meier life-table analysis), complication rates, and risk factors of surgical failure (multivariate analysis via Cox proportional hazard ratio [HR] model). Surgical success (with or without medication use) was defined as an IOP between 6 and $15 \mathrm{mmHg}$ with a postoperative IOP reduction of at least $20 \%$ (criterion A) or an IOP between 6 and $12 \mathrm{mmHg}$ with a postoperative IOP reduction of at least $30 \%$ (criterion B). A surgical failure was defined as not meeting success criteria at two consecutive follow-up visits at least 1 month after surgery or the need for additional glaucoma surgery. The IOP levels later than 1 month were used as determinants of surgical success so that short-term IOP fluctuations would not influence long-term surgical success rates. Risk factors of surgical failure were identified using the Cox proportional HR model with multivariate analysis. The variables for multivariate analysis were selected from variables with a $P$-value of $<0.3$, as determined by univariate analysis. Variables tested included age, sex, preoperative IOP, number of anti-glaucoma medications, glaucoma type, lens status, past intraocular surgeries, selective laser trabeculoplasty, and the phacoemulsification combination procedure.

\section{Procedure safety}

Safety measures included intra- and postoperative complication rates and BCVA 2 years after surgery. Examined postoperative complications included hyphema, shallow chamber, wound leaks, IOP spikes (IOP $\geq 30 \mathrm{mmHg}$ within 1 month of 360S-LOT ab interno), hypotony (IOP $\leq 5 \mathrm{mmHg}$ ), infection, decreased corneal endothelial cell density, and vision loss.

\section{Statistical analyses}

The JMP statistical package (version 13; SAS Institute Inc., Cary, NC, USA) was used to perform all data analyses. Continuous data were expressed as mean \pm SD and were examined using paired $t$-tests and Wilcoxon signed-rank tests as appropriate. Categorical data were expressed as n (\%) and were examined using Fisher's exact test. The Kaplan-Meier survival curve analysis was used to evaluate the cumulative probability of success, and a log-rank test was used for comparison of the $360 \mathrm{~S}-\mathrm{LOT}$ ab interno procedure and the combined 360S-LOT ab interno/phacoemulsification procedure. Statistical significance was defined as $P<0.05$.

\section{Results}

\section{Baseline patient characteristics}

The 360S-LOT ab interno procedure was performed on 83 eyes between March 2014 and September 2015. In all, 19 patients were excluded because they did not have OAG ( 9 eyes) or had already had the fellow eye included in this study (10 eyes). In the overall study, 14 of 64 (22\%) eyes were lost to follow-up by 2 years; 50 eyes of 50 patients ( 20 males, $73.5 \pm 9.2$ years old) reached the 2 -year point. Preoperative ocular characteristics and treatment information of patients are summarized in Table 1 . The 360 S-LOT ab interno procedure was performed alone in 19 (29.7\%) eyes and in combination with phacoemulsification in 45 (70.3\%) eyes.

\section{Outcomes}

In 50 eyes of 50 patients that underwent follow-up for 2 years, surgery significantly reduced IOP from $18.4 \pm 2.9 \mathrm{mmHg}$ to $13.4 \pm 3.5 \mathrm{mmHg}$ after 1 year $(27.2 \%$ reduction $)$ and to $13.4 \pm$ $3.0 \mathrm{mmHg}$ after 2 years $(27.2 \%$ reduction, both $P<0.001$; Table 2 and Figure 1). In eyes that underwent the 360S-LOT $\mathrm{ab}$ interno alone procedure, IOP was significantly reduced from $19.1 \pm 2.2 \mathrm{mmHg}$ before surgery to $14.9 \pm 3.0 \mathrm{mmHg}$ 1 year after surgery $(22.0 \%$ reduction $)$ and $14.6 \pm 2.9 \mathrm{mmHg}$ 2 years after surgery $(23.6 \%$ reduction, both $P<0.001)$. In eyes that underwent a combined 360S-LOT ab interno/phacoemulsification procedure, IOP was significantly reduced from $18.1 \pm 3.2 \mathrm{mmHg}$ before surgery to $12.7 \pm 3.6 \mathrm{mmHg}$ 1 year after surgery (29.3\% reduction) and $12.7 \pm 2.9 \mathrm{mmHg}$ 2 years after surgery $(28.5 \%$ reduction, both $P<0.001)$.

The number of anti-glaucoma medications used was $1.8 \pm 1.5$ before surgery and $0.9 \pm 1.2(P<0.001)$ and $1.3 \pm 1.5(P=0.101) 1$ and 2 years after surgery, respectively. In eyes that underwent the $360 \mathrm{~S}-\mathrm{LOT}$ ab interno procedure alone, the number of anti-glaucoma medications used was also significantly reduced from $3.3 \pm 0.8$ before surgery to $1.7 \pm 1.3(P<0.001)$ and $2.2 \pm 1.4(P=0.001) 1$ and 2 years after surgery, respectively. In eyes that underwent a combined 360S-LOT ab interno/phacoemulsification procedure, the number of anti-glaucoma medications was significantly reduced from $1.0 \pm 1.2$ before surgery to $0.4 \pm$ 0.91 year after surgery $(P=0.021)$. However, the number of anti-glaucoma medications used 2 years after surgery was 
Table I Preoperative demographic and ocular characteristics of patients undergoing 360S-LOT ab interno

\begin{tabular}{|c|c|c|c|c|}
\hline Characteristics & All patients & $\begin{array}{l}\text { 360S-LOT ab } \\
\text { interno }\end{array}$ & $\begin{array}{l}\text { 360S-LOT ab interno } \\
\text { with PEA + IOL }\end{array}$ & $P$-value \\
\hline $\mathrm{n}$, patients/eyes & $64 / 64$ & $19 / 19$ & $45 / 45$ & NA \\
\hline Age, years & $73.9 \pm 8.7(55-90)$ & $72.4 \pm 8.3(59-90)$ & $74.5 \pm 8.9(55-90)$ & 0.396 \\
\hline Male/female & $27 / 37$ & $7 / 12$ & $20 / 25$ & 0.390 \\
\hline Preoperative IOP, mmHg & $\mid 8.3 \pm 3.4(||-3 \mid)$ & $18.9 \pm 2.6(13-24)$ & $|8.| \pm 3.2(||-3 \mid)$ & 0.376 \\
\hline Number of medications & $1.6 \pm 1.5(0-4)$ & $3.3 \pm 0.7(2-4)$ & $0.9 \pm 1.2(0-4)$ & $<0.001$ \\
\hline Phakic/pseudophakic & $58 / 6$ & $13 / 6$ & $45 / 0$ & $<0.001$ \\
\hline LogMAR BCVA & $0.28 \pm 0.35$ & $0.06 \pm 0.16$ & $0.38 \pm 0.37$ & 0.001 \\
\hline Visual field MD, dB & $-9.8 \pm 6.7$ & $-11.0 \pm 6.3$ & $-9.3 \pm 6.9$ & 0.358 \\
\hline Corneal endothelial cell density, cells $/ \mathrm{mm}^{2}$ & $2,578.1 \pm 303.6$ & $2,500.1 \pm 344.9$ & $2,609.3 \pm 283.5$ & 0.200 \\
\hline Type of glaucoma, n (\%) & & & & 0.046 \\
\hline POAG & $28(44)$ & II (58) & $17(38)$ & \\
\hline NTG & $20(3 I)$ & $2(I I)$ & $18(40)$ & \\
\hline XFG & $16(25)$ & $6(32)$ & $10(22)$ & \\
\hline Previous surgical or laser treatment, n (\%) & $10(16)$ & $9(47)$ & $I(2)$ & $<0.001$ \\
\hline Cataract extraction & $6(9)$ & $6(32)$ & $0(0)$ & \\
\hline Scleral buckle & I (2) & $0(0)$ & I (2) & \\
\hline Selective laser trabeculoplasty & $3(5)$ & $3(16)$ & $0(0)$ & \\
\hline
\end{tabular}

Note: Continuous data are expressed as mean \pm SD (range).

Abbreviations: 360S-LOT, 360-degree suture trabeculotomy; PEA + IOL, phacoemulsification with intraocular lens implant; NA, not applicable; IOP, intraocular pressure; logMAR, logarithm of the minimum angle of resolution; BCVA, best-corrected visual acuity; MD, mean deviation; POAG, primary open-angle glaucoma; NTG, normal tension glaucoma; XFG, exfoliation glaucoma.

$0.8 \pm 1.3$, which was not significantly different from that before surgery $(P=0.420)$.

The surgical success rate, based on an IOP of $<15 \mathrm{mmHg}$, which was set as the postoperative target with or without medication use, was $64.2 \%$. Figure 2 shows surgical success, using the Kaplan-Meier survival analysis, for both criteria $A$ and B. Two years after 360S-LOT ab interno, the surgical success rate was $49.2 \%$ for criterion $A$ and $16.0 \%$ for criterion B. Although the success rate of the combined 360S-LOT ab interno/phacoemulsification procedure was significantly

Table 2 IOP and number of anti-glaucoma medications used in patients who underwent 360S-LOT ab interno

\begin{tabular}{|c|c|c|c|c|}
\hline & All patients & $\begin{array}{l}360 S-L O T \\
\text { ab interno } \\
\text { alone }\end{array}$ & $\begin{array}{l}360 \text { S-LOT ab } \\
\text { interno with } \\
\text { PEA + IOL }\end{array}$ & $P$-value \\
\hline $\mathrm{n}$, patients/eyes & $50 / 50$ & $17 / 17$ & $33 / 33$ & NA \\
\hline \multicolumn{5}{|l|}{ IOP (mmHg) } \\
\hline Before surgery & $18.4 \pm 2.9$ & $19.1 \pm 2.2$ & $18.1 \pm 3.2$ & 0.228 \\
\hline I day & $17.8 \pm 11.3$ & $15.7 \pm 6.4$ & $18.9 \pm 13.1$ & 0.176 \\
\hline I week & $16.6 \pm 8.0$ & $19.4 \pm 9.0$ & $15.2 \pm 7.1$ & 0.078 \\
\hline I month & $13.5 \pm 3.3$ & $|5| \pm 4.0$. & $12.7 \pm 2.6$ & 0.012 \\
\hline 3 months & $13.3 \pm 3.1$ & $15.2 \pm 3.4$ & $12.3 \pm 2.5$ & 0.002 \\
\hline 6 months & $13.0 \pm 3.7$ & $\mid 4.6 \pm 4.1$ & $12.1 \pm 3.1$ & 0.022 \\
\hline 12 months & $13.4 \pm 3.5$ & $14.9 \pm 3.0$ & $12.7 \pm 3.6$ & 0.034 \\
\hline 24 months & $13.4 \pm 3.0$ & $14.6 \pm 2.9$ & $12.7 \pm 2.9$ & 0.032 \\
\hline I2-month IOP reduction & $26.7 \% \pm 17.6 \%$ & $21.6 \% \pm 15.8 \%$ & $29.3 \% \pm 18.2 \%$ & 0.151 \\
\hline 24-month IOP reduction & $26.9 \% \pm 15.3 \%$ & $23.0 \% \pm 14.7 \%$ & $28.9 \% \pm 15.5 \%$ & 0.200 \\
\hline \multicolumn{5}{|c|}{ Number of anti-glaucoma medications } \\
\hline Before surgery & $1.8 \pm 1.5$ & $3.3 \pm 0.8$ & $1.0 \pm 1.2$ & $<0.001$ \\
\hline 12 months & $0.9 \pm 1.2$ & $1.7 \pm 1.3$ & $0.4 \pm 0.9$ & $<0.001$ \\
\hline 24 months & $1.3 \pm 1.5$ & $2.2 \pm 1.4$ & $0.8 \pm 1.3$ & 0.002 \\
\hline
\end{tabular}

Notes: The IOP and number of anti-glaucoma medications are expressed as mean \pm standard deviation. $P$-value for the IOP calculated using a paired $t$-test. $P$-value for number of anti-glaucoma medications calculated using a Wilcoxon signed-rank test.

Abbreviations: IOP, intraocular pressure; 360S-LOT, 360-degree suture trabeculotomy; PEA + IOL, phacoemulsification with intraocular lens implant; NA, not applicable. 


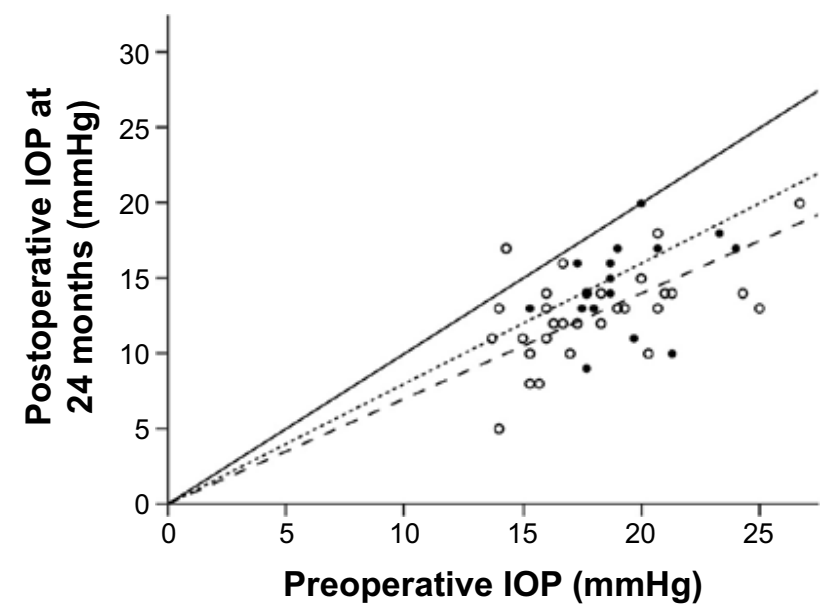

Figure I Plot of postoperative IOP at 24 months versus preoperative IOP in eyes that underwent the 360S-LOT ab interno procedure with $(O)$ and without $(\bullet)$ PEA + IOL.

Notes: Most eyes had a reduction in IOP following surgery regardless of the procedure performed. Lines representing no IOP change (-), a $20 \%$ IOP reduction (....), and a 30\% IOP reduction (----) are shown for reference.

Abbreviations: IOP, intraocular pressure; 360S-LOT, 360-degree suture trabeculotomy; PEA + IOL, phacoemulsification with intraocular lens implant.

higher than that of the 360S-LOT ab interno alone procedure in criterion $\mathrm{A}(P=0.043)$, there was no significant difference between both groups in criterion $\mathrm{B}(P=0.070)$.

Univariate analysis (Table 3 ) indicated that for condition $\mathrm{A}, \geq 2$ anti-glaucoma medications and 360S-LOT $\mathrm{ab}$ interno alone were significant risk factors of surgical failure $(P=0.027$ and $P=0.043$, respectively). Variables with $P$-values of $<0.3$ were age, female sex, and past history ( $P=0.087, P=0.110$, and $P=0.053$, respectively). Although univariate analysis showed that no variable was a significant risk factor of surgical failure for criterion $\mathrm{B}$, variables with $P$-values of $<0.3$ were age, $\geq 2$ anti-glaucoma

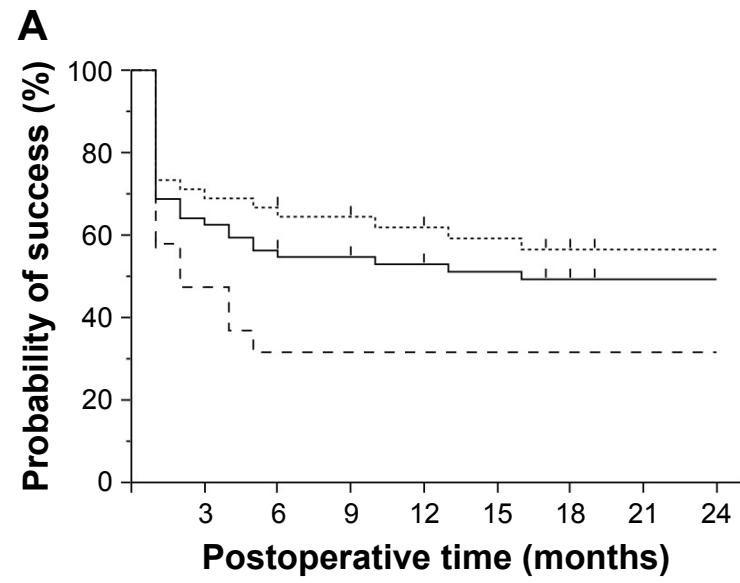

medications, past history, and 360S-LOT ab interno alone $(P=0.232, P=0.165, P=0.247$, and $P=0.070$, respectively). Multivariate analysis (Cox proportional HR model) did not reveal any factors that were predictive of surgical success or failure (Tables 4 and 5).

\section{Safety}

No patient experienced any severe complications during surgery or the 2-year follow-up period. Additionally, no significant safety differences were observed between eyes that underwent 360S-LOT ab interno alone and those that underwent a combined 360S-LOT ab interno/phacoemulsification procedure (Table 6). It should be noted that 25 (50.0\%) eyes had a hyphema 1 day after surgery. The hyphema spontaneously resolved by 1 month in all but two eyes, which required anterior chamber washing at 1 week because the hyphema was massive and was causing IOP spikes. In addition to these two eyes, 12 other eyes also experienced IOP spikes ( 14 eyes in total [28\%]) within the first month after surgery. In these 12 eyes, a temporary systemic carbonic anhydrase inhibitor was used to lower IOP, which returned to within the normal range within 1 week.

The endothelial cell density was $2,460.1 \pm 361.0$ cells $/ \mathrm{mm}^{2}$ after 2 years of surgery, a $4.9 \% \pm 7.6 \%$ reduction from that before surgery. One ( $2 \%)$ eye required a trabeculectomy because of uncontrolled IOP 22 months after surgery. In the group that underwent combined 360S-LOT ab interno/ phacoemulsification procedure, logMAR BCVA significantly improved from $0.34 \pm 0.36$ (Snellen equivalent: $20 / 44$ ) before surgery to $0.14 \pm 0.26$ (Snellen equivalent: $20 / 28$ ) 2 years after surgery $(P=0.001)$. Eyes that underwent 360 S-LOT ab

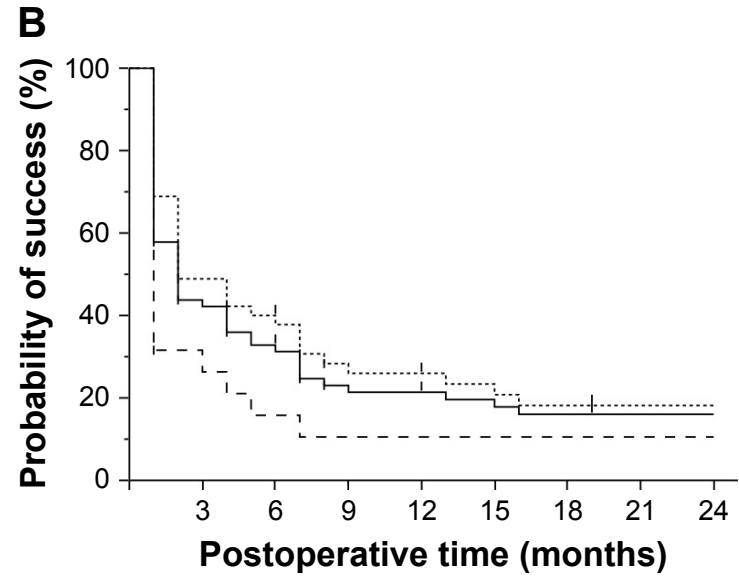

Figure 2 Kaplan-Meier survival curves for surgical success using (A) criterion A (IOP between 6 and I5 mmHg and IOP reduction $\geq 20 \%$ ) and (B) criterion B (IOP between 6 and $12 \mathrm{mmHg}$ and IOP reduction $\geq 30 \%$ ).

Notes: Criteria were applied independent of anti-glaucoma medication use. Two-years after surgery, the success rates using criteria $A$ and $B$ were $49.2 \%$ and $16.0 \%$, respectively. Lines representing all patients (-) and 360S-LOT ab interno with (....) and without (----) PEA + IOL are shown for reference. Abbreviations: IOP, intraocular pressure; 360S-LOT, 360-degree suture trabeculotomy; PEA + IOL, phacoemulsification with intraocular lens implant. 
Table 3 Univariable analysis of risk factors for 360S-LOT ab interno

\begin{tabular}{|c|c|c|c|c|c|}
\hline \multirow[t]{2}{*}{ Variable } & \multirow[t]{2}{*}{$\begin{array}{l}\text { No. of } \\
\text { eyes }\end{array}$} & \multicolumn{2}{|c|}{$\begin{array}{l}\text { Criterion A for surgical } \\
\text { success }\end{array}$} & \multicolumn{2}{|c|}{$\begin{array}{l}\text { Criterion B for surgical } \\
\text { success }\end{array}$} \\
\hline & & $\begin{array}{l}\text { 2-Year } \\
\text { probability } \\
\text { of success }\end{array}$ & $P$-value & $\begin{array}{l}\text { 2-Year } \\
\text { probability } \\
\text { of success }\end{array}$ & $P$-value \\
\hline Age (years) & & & 0.087 & & 0.232 \\
\hline$>75$ & 31 & 60.7 & & 27.4 & \\
\hline$\leq 75$ & 33 & 38.3 & & 6.1 & \\
\hline Preoperative IOP (mmHg) & & & 0.644 & & 0.702 \\
\hline$>18$ & 32 & 52.5 & & 20.3 & \\
\hline$\leq 18$ & 32 & 46.3 & & 12.5 & \\
\hline Number of anti-glaucoma medications & & & 0.027 & & 0.165 \\
\hline$\geq 2$ & 33 & 36.1 & & 15.2 & \\
\hline$<2$ & 31 & 63.5 & & 17.5 & \\
\hline Sex & & & 0.110 & & 0.352 \\
\hline Male & 27 & 62.5 & & 19.6 & \\
\hline Female & 37 & 40.1 & & 13.5 & \\
\hline Type of glaucoma & & & 0.300 & & 0.520 \\
\hline POAG & 28 & 39 & & 13.4 & \\
\hline NTG & 20 & 58.2 & & 11.4 & \\
\hline XFG & 16 & 55.6 & & 25 & \\
\hline Lens status & & & 0.465 & & 0.618 \\
\hline Phakia & 58 & 50.8 & & 14.1 & \\
\hline Pseudophakia & 6 & 33.3 & & 33.3 & \\
\hline Past history & & & 0.053 & & 0.247 \\
\hline None & 55 & 53.5 & & 14.8 & \\
\hline Intraocular surgeries & 7 & 28.6 & & 28.6 & \\
\hline Selective laser trabeculoplasty & 2 & 0 & & 0 & \\
\hline With/without PEA + IOL & & & 0.043 & & 0.070 \\
\hline 360S-LOT ab interno alone & 19 & 31.6 & & 10.5 & \\
\hline 360S-LOT ab interno with PEA + IOL & 45 & 56.4 & & 18.2 & \\
\hline
\end{tabular}

Note: $P$-values were based on log-rank test.

Abbreviations: 360S-LOT, 360-degree suture trabeculotomy; IOP, intraocular pressure; POAG, primary open-angle glaucoma; NTG, normal tension glaucoma; XFG, exfoliation glaucoma; PEA + IOL, phacoemulsification with intraocular lens implant.

Table 4 Multivariate analysis using the Cox proportional HR model analysis for 360S-LOT ab interno (criterion A)

\begin{tabular}{llll}
\hline Variable & \multicolumn{3}{l}{$\begin{array}{l}\text { Criterion A for surgical } \\
\text { success }\end{array}$} \\
\cline { 2 - 4 } & $\begin{array}{l}\text { Risk } \\
\text { ratio }\end{array}$ & $95 \%$ Cl & P-value \\
\hline Age (years) & 0.97 & $0.94-1.01$ & 0.143 \\
Number of anti-glaucoma medications & 1.34 & $0.98-1.83$ & 0.077 \\
Sex & & & 0.255 \\
$\quad$ Male & $\mathrm{I}$ & & \\
$\quad$ Female & $\mathrm{I} .57$ & $0.73-3.58$ & \\
Past history & & & 0.560 \\
$\quad$ None & $\mathrm{I}$ & & \\
$\quad$ Intraocular surgeries & $\mathrm{I} .66$ & $0.50-4.76$ & 0.383 \\
$\quad$ Selective laser trabeculoplasty & 1.94 & $0.28-8.25$ & 0.444 \\
With/without PEA + IOL & & & 0.560 \\
$\quad$ 360S-LOT ab interno alone & $\mathrm{I}$ & & \\
360S-LOT ab interno with PEA + IOL & $\mathrm{I} .38$ & $0.47-3.92$ & 0.546 \\
\hline
\end{tabular}

Note: $P$-values are based on Cox proportional HR models.

Abbreviations: HR, hazard ratio; 360S-LOT, 360-degree suture trabeculotomy; PEA + IOL, phacoemulsification with intraocular lens implant. interno alone did not have a significant change in BCVA 2 years following surgery (preoperative: $0.06 \pm 0.17$ [Snellen equivalent: 20/23], postoperative: $0.11 \pm 0.15$ [Snellen equivalent: 20/26]; $P=0.073$ ).

\section{Discussion}

This prospective study evaluated the 2-year efficacy of the 360S-LOT ab interno procedure performed to treat OAG. Risk factors of surgical failure were also examined. The 360S-LOT ab interno procedure significantly reduced IOP at both the 1- and 2-year time points compared to the baseline values. No prognostic factors for 360S-LOT ab interno failure were identified by the Cox proportional HR model analysis.

Prior studies have examined the efficacy of a modified 360S-LOT ab externo procedure. One year following surgery, IOP was $10.9 \mathrm{mmHg}$ in eyes with $\mathrm{XFG}^{11}$ and 
Table 5 Multivariate analysis using the Cox proportional HR model analysis for 360S-LOT ab interno (criterion B)

\begin{tabular}{llll}
\hline Variable & \multicolumn{3}{l}{$\begin{array}{l}\text { Criteria B for surgical } \\
\text { success }\end{array}$} \\
\cline { 2 - 4 } & $\begin{array}{l}\text { Risk } \\
\text { ratio }\end{array}$ & $95 \%$ Cl & P-value \\
& 0.97 & $0.94-1.00$ & 0.060 \\
Age (years) & 1.09 & $0.84-1.39$ & 0.505 \\
Number of anti-glaucoma medications & & & 0.604 \\
Past history & $\mathrm{I}$ & & \\
$\quad$ None & 0.69 & $0.22-1.83$ & 0.467 \\
$\quad$ Intraocular surgeries & $\mathrm{I} .57$ & $0.24-6.04$ & 0.584 \\
$\quad$ Selective laser trabeculoplasty & & & 0.636 \\
With/without PEA + IOL & $\mathrm{I}$ & & \\
$\quad$ 360S-LOT ab interno alone & & & \\
360S-LOT ab interno with PEA + IOL & 0.80 & $0.32-1.98$ & 0.637
\end{tabular}

Note: $P$-values are based on Cox proportional HR models.

Abbreviations: HR, hazard ratio; 360S-LOT, 360-degree suture trabeculotomy; PEA + IOL, phacoemulsification with intraocular lens implant.

14.5 mmHg in eyes with OAG. ${ }^{12}$ Two years following surgery, IOP was $\sim 13 \mathrm{mmHg}$ in eyes with OAG. ${ }^{13}$ Additionally, Grover et $\mathrm{al}^{14}$ found that gonioscopy-assisted transluminal trabeculotomy, which includes creating a 360-degree trabeculotomy with a thermally blunted suture or microcatheter, reduced IOP to $12.4-16.2 \mathrm{mmHg} 1$ year after surgery and $\sim 16 \mathrm{mmHg} 2$ years after surgery. ${ }^{15}$ This was even true in patients who had undergone prior incisional glaucoma surgery. Our results with the 360S-LOT ab interno procedure were slightly superior to those of the previous study by Grover et al, where an IOP of $13.4 \mathrm{mmHg}$ was observed at 2 years after surgery. We suspect that, in the present study, the preoperative IOP was lower and the patients had no previous glaucoma surgery, in contrast with the prior study.
However, the postoperative IOP in the present study was $13.4 \mathrm{mmHg}$ at 2 years after surgery, higher than the episcleral venous pressure, despite nearly elimination of the resistance of the inner layer of the circumferential Schlemm's canal. Episcleral venous pressures in POAG and NTG patients have been reported as $12.1 \pm 0.5 \mathrm{mmHg}$ and $11.6 \pm 0.4 \mathrm{mmHg}$, respectively, both of which are significantly higher than the episcleral venous pressure of normal eyes $(9.5 \pm 0.2 \mathrm{mmHg}){ }^{9}$ Thus, we suspect that resistance may be increased by inflammation or coagula occurring in the early postoperative period or by trabecular meshwork and Schlemm's canal inner layer incision closure during the wound healing process. ${ }^{16}$

Nearly half (45.8\%) of patients in the current study had a postoperative IOP of $\leq 15 \mathrm{mmHg}$ and a postoperative IOP reduction of at least $20 \% 2$ years after surgery. Therefore, the 360S-LOT ab interno procedure may be a useful option for treating mild- to moderate-stage OAG. However, because only $16.0 \%$ of patients had an IOP of $\leq 12 \mathrm{mmHg}$ with a postoperative IOP reduction of $\geq 30 \%$, this procedure is likely not useful for treating late-stage glaucoma. Our mid-term success rates with the ab interno procedure were likely similar or better than those previously reported for standard trabeculotomy (ab externo). Success rates of the ab externo procedure, which involves incising the trabecular meshwork and Schlemm's canal inner layer at $120^{\circ}$, decreased over the 5 years following surgery. ${ }^{16}$ This efficacy decrease over time was likely caused by trabecular meshwork and Schlemm's canal inner layer incision closure that occurred during the wound healing process. It is possible that the wider trabecular meshwork and Schlemm's canal inner layer opening that is made with the

Table 6 Intraoperative and postoperative complications following 360S-LOT ab interno

\begin{tabular}{|c|c|c|c|c|}
\hline & All patients & $\begin{array}{l}\text { 360S-LOT ab } \\
\text { interno alone }\end{array}$ & $\begin{array}{l}\text { 360S-LOT ab } \\
\text { interno + PEA + IOL }\end{array}$ & $P$-value \\
\hline $\mathrm{n}$, patients/eyes & $50 / 50$ & $17 / 17$ & $33 / 33$ & NA \\
\hline Intraoperative blood reflux, n (\%) & $50(100)$ & $17(100)$ & $33(100)$ & 1.000 \\
\hline Hyphema, n (\%) & & & & 0.072 \\
\hline Day I & $25(50)$ & $12(7 \mid)$ & $13(39)$ & \\
\hline Week I & $12(24)$ & $7(4 I)$ & $5(15)$ & \\
\hline Week 2 & $4(8)$ & $2(12)$ & $2(6)$ & \\
\hline Shallow chamber & $0(0)$ & $0(0)$ & $0(0)$ & \\
\hline Wound leaks & $0(0)$ & $0(0)$ & $0(0)$ & 1.000 \\
\hline IOP spike (>30 mmHg), n (\%) & $14(28)$ & $4(24)$ & $10(36)$ & 0.755 \\
\hline Hypotony (IOP < $5 \mathrm{mmHg}$ ) & $0(0)$ & $0(0)$ & $0(0)$ & 1.000 \\
\hline Infection & $0(0)$ & $0(0)$ & $0(0)$ & 1.000 \\
\hline Corneal endothelial cell density reduction & $4.9 \% \pm 7.6 \%$ & $5.2 \% \pm 5.0 \%$ & $4.8 \% \pm 8.6 \%$ & 0.859 \\
\hline Anterior chamber washing out, $\mathrm{n}(\%)$ & $2(4)$ & I (6) & I (3) & 1.000 \\
\hline Subsequent glaucoma surgery, $\mathrm{n}(\%)$ & I (2) & I (6) & $0(0)$ & 0.340 \\
\hline
\end{tabular}

Note: Corneal endothelial cell density reduction is expressed as mean $\pm \mathrm{SD}$.

Abbreviations: 360S-LOT, 360-degree suture trabeculotomy; PEA + IOL, phacoemulsification with intraocular lens implant; NA, not applicable; IOP, intraocular pressure. 
360-degree incision has a lower closure rate than the opening made with the 120-degree incision. On the other hand, Manabe et $\mathrm{al}^{12}$ reported that the 150-degree or more extent of the incision in the Schlemm's canal during S-LOT ab externo did not affect the reduction in IOP at 1 year postoperatively. We are currently prospectively investigating the relationship between Schlemm's canal incision width and the postoperative IOP reduction, because there is a possibility that 360-degree incision in the Schlemm's canal is too invasive.

Although no prognostic factors for 360S-LOT ab interno failure were identified by the Cox proportional HR model, univariate analysis by the Kaplan-Meier survival analysis method revealed that $\geq 2$ anti-glaucoma medications and 360S-LOT $\mathrm{ab}$ interno alone were both significant risk factors of surgical failure. Additionally, the IOP was significantly lower in the 360S-LOT ab interno with phacoemulsification group than in the 360S-LOT ab interno group throughout the follow-up period, 1 month after surgery, and the success rate of the combined 360S-LOT ab interno/phacoemulsification procedure was significantly higher than that of the 360S-LOT ab interno alone procedure in criterion A. Although the phacoemulsification procedure alone reduces IOP in glaucomatous eyes, ${ }^{17,18}$ an earlier report discussed the difficulties of separating the effects of phacoemulsification and glaucoma surgeries because the two procedures have different goals. ${ }^{19}$ Many patients who require 360S-LOT ab interno alone have uncontrolled IOP even though they are using anti-glaucoma medications. In these patients, the surgical goal is to lower IOP. However, patients who undergo 360S-LOT ab interno in combination with phacoemulsification procedure generally have a visually significant cataract but controlled IOP. These patients have a surgical goal of increasing vision (from cataract removal) and reducing the number of anti-glaucoma medications.

No serious complications (eg, shallow anterior chamber, choroidal detachment, infection, or wound leakage) occurred in any of our patients. The most frequent postoperative complications observed were hyphema and IOP spikes, but the incidences of hyphema and IOP spikes were not particularly high, and in most eyes, these complications were easily controlled. Therefore, the $360 \mathrm{~S}-L O T$ ab interno is likely a safe procedure, but careful follow-up is needed within the first postoperative month.

The current study has several limitations. First, our sample size was small. Second, this study was not controlled. Third, the mean preoperative IOP was $<21 \mathrm{mmHg}$, which is not excessively high. The Tajimi Study in Japan reported a POAG prevalence of $3.6 \%$ in patients with an IOP of $\leq 21 \mathrm{mmHg}$, which was significantly higher than the POAG prevalence of $0.3 \%$ in patients with an IOP of $>21 \mathrm{mmHg} .{ }^{20}$ Accordingly, there are many cases in which treatments are performed in patients with OAG but not a high IOP in Japan, similar to that found in this study. Because of these limitations, additional research is needed on a larger group of patients to better understand long-term efficacy of the $360 \mathrm{~S}-\mathrm{LOT}$ ab interno procedure.

\section{Conclusion}

The current study demonstrates that the 360S-LOT ab interno procedure is likely a safe and effective treatment for OAG over the midterm (at least 2 years). Thus, this procedure should be considered for surgically managing mild to moderate OAG. It should be noted that further studies are needed to validate our findings.

\section{Disclosure}

The authors report no conflicts of interest in this work.

\section{References}

1. Tanihara H, Negi A, Akimoto M, Nagata M. Long-term surgical results of combined trabeculotomy ab externo and cataract extraction. Ophthalmic Surg. 1995;26(4):316-324.

2. Wada Y, Nakatsu A, Kondo T. Long-term results of trabeculotomy ab externo. Ophthalmic Surg. 1994;25(5):317-320.

3. Chihara E, Nishida A, Kodo M, et al. Trabeculotomy ab externo: an alternative treatment in adult patients with primary open-angle glaucoma. Ophthalmic Surg. 1993;24(11):735-739.

4. Hoffmann E, Schwenn O, Karallus M, Krummenauer F, Grehn F, Pfeiffer N. Long-term results of cataract surgery combined with trabeculotomy. Graefes Arch Clin Exp Ophthalmol. 2002;240(1):2-6.

5. Mizoguchi T, Kuroda S, Terauchi H, Nagata M. Trabeculotomy combined with phacoemulsification and implantation of intraocular lens for primary open-angle glaucoma. Semin Ophthalmol. 2001;16(3): 162-167.

6. Gimbel HV, Meyer D. Small incision trabeculotomy combined with phacoemulsification and intraocular lens implantation. J Cataract Refract Surg. 1993;19(1):92-96.

7. Chin S, Nitta T, Shinmei Y, et al. Reduction of intraocular pressure using a modified 360-degree suture trabeculotomy technique in primary and secondary open-angle glaucoma: a pilot study. J Glaucoma. 2012; 21(6):401-407.

8. Sato T, Hirata A, Mizoguchi T. Outcomes of 360 degrees suture trabeculotomy with deep sclerectomy combined with cataract surgery for primary open angle glaucoma and coexisting cataract. Clin Ophthalmol. 2014;8:1301-1310.

9. Selbach JM, Posielek K, Steuhl KP, Kremmer S. Episcleral venous pressure in untreated primary open-angle and normal-tension glaucoma. Ophthalmologica. 2005;219(6):357-361.

10. Sato T, Hirata A, Mizoguchi T. Prospective, noncomparative, nonrandomized case study of short-term outcomes of 360 degrees suture trabeculotomy ab interno in patients with open-angle glaucoma. Clin Ophthalmol. 2015;9:63-68.

11. Hepsen IF, Guler E, Yalcin NG, Kumova D, Aktas ZP. Modified 360degree suture trabeculotomy for pseudoexfoliation glaucoma: 12-month results. J Glaucoma. 2016;25(4):e408-e412.

12. Manabe SI, Sawaguchi S, Hayashi K. The effect of the extent of the incision in the Schlemm canal on the surgical outcomes of suture trabeculotomy for open-angle glaucoma. Jpn J Ophthalmol. 2017; 61(1):99-104. 
13. Shinmei Y, Kijima R, Nitta T, et al. Modified 360-degree suture trabeculotomy combined with phacoemulsification and intraocular lens implantation for glaucoma and coexisting cataract. J Cataract Refract Surg. 2016;42(11):1634-1641.

14. Grover DS, Godfrey DG, Smith O, Feuer WJ, Montes de Oca I, Fellman RL. Gonioscopy-assisted transluminal trabeculotomy, ab interno trabeculotomy: technique report and preliminary results. Ophthalmology. 2014;121(4):855-861.

15. Grover DS, Godfrey DG, Smith O, Shi W, Feuer WJ, Fellman RL. Outcomes of gonioscopy-assisted transluminal trabeculotomy (GATT) in eyes with prior incisional glaucoma surgery. J Glaucoma. 2017;26(1): 41-45.

16. Tanihara H, Negi A, Akimoto M, et al. Surgical effects of trabeculotomy ab externo on adult eyes with primary open angle glaucoma and pseudoexfoliation syndrome. Arch Ophthalmol. 1993;111(12):1653-1661.
17. Chen PP, Lin SC, Junk AK, Radhakrishnan S, Singh K, Chen TC. The effect of phacoemulsification on intraocular pressure in glaucoma patients: a report by the American Academy of Ophthalmology. Ophthalmology. 2015;122(7):1294-1307.

18. Hayashi K, Hayashi H, Nakao F, Hayashi F. Effect of cataract surgery on intraocular pressure control in glaucoma patients. J Cataract Refract Surg. 2001;27(11):1779-1786.

19. Ahuja Y, Ma Khin Pyi S, Malihi M, Hodge DO, Sit AJ. Clinical results of $\mathrm{ab}$ interno trabeculotomy using the trabectome for openangle glaucoma: the Mayo Clinic series in Rochester, Minnesota. Am J Ophthalmol. 2013;156(5):927.e-935.e.

20. Iwase A, Suzuki Y, Araie M, et al. The prevalence of primary openangle glaucoma in Japanese: the Tajimi Study. Ophthalmology. 2004 111(9):1641-1648.
Clinical Ophthalmology

\section{Publish your work in this journal}

Clinical Ophthalmology is an international, peer-reviewed journa covering all subspecialties within ophthalmology. Key topics include: Optometry; Visual science; Pharmacology and drug therapy in eye diseases; Basic Sciences; Primary and Secondary eye care; Patien Safety and Quality of Care Improvements. This journal is indexed on

Submit your manuscript here: http://www.dovepress.com/clinical-ophthalmology-journal

\section{Dovepress}

PubMed Central and CAS, and is the official journal of The Society of Clinical Ophthalmology (SCO). The manuscript management system is completely online and includes a very quick and fair peer-review system, which is all easy to use. Visit http://www.dovepress.com/ testimonials.php to read real quotes from published authors. 